\title{
The rise of methicillin resistant Staphylococcus aureus: now the dominant cause of skin and soft tissue infection in Central Australia
}

\author{
E. MACMORRAN ${ }^{1,2 *}$, S. HARCH ${ }^{1,2}, \mathrm{EATHAN}^{2,3}, \mathrm{~S} \mathrm{LANE}^{2,3}, \mathrm{STONG}^{4,5}$, \\ L CRAWFORD $^{1}$, S KRISHNASWAMY ${ }^{1}$ AND S HEWAGAMA ${ }^{1}$ \\ ${ }^{1}$ Alice Springs Hospital, Northern Territory, Australia \\ ${ }^{2}$ Infectious Diseases, Barwon Health, Geelong, Victoria, Australia \\ ${ }^{3}$ Deakin University, Burwood, Victoria, Australia \\ ${ }^{4}$ Victorian Infectious Diseases Service, The Royal Melbourne Hospital, and the University of Melbourne at the \\ Peter Doherty Institute for Infection and Immunity, Victoria, Australia \\ ${ }^{5}$ Menzies School of Health Research, Darwin, Australia
}

Received 25 March 2017; Final revision 8 June 2017; Accepted 13 July 2017;

first published online 14 August 2017

\section{SUMMARY}

This study aimed to examine the epidemiology and treatment outcomes of community-onset purulent staphylococcal skin and soft tissue infections (SSTI) in Central Australia. We performed a prospective observational study of patients hospitalised with community-onset purulent staphylococcal SSTI $(n=160)$. Indigenous patients accounted for $78 \%$ of cases. Patients were predominantly young adults; however, there were high rates of co-morbid disease. Communityassociated methicillin-resistant Staphylococcus aureus (CA-MRSA) was the dominant phenotype, accounting for $60 \%$ of cases. Hospitalisation during the preceding 6 months, and haemodialysis dependence were significant predictors of CA-MRSA infection on univariate analysis. Clinical presentation and treatment outcomes were found to be comparable for methicillin-susceptible $S$. aureus (MSSA) and methicillin-resistant cases. All MRSA isolates were characterised as nonmulti-resistant, with this term used interchangeably with CA-MRSA in this analysis. We did not find an association between receipt of an active antimicrobial agent within the first $48 \mathrm{~h}$, and progression of infection; need for further surgical debridement; unplanned General Practitioner or hospital re-presentation; or need for further antibiotics. At least one adverse outcome was experienced by $39 \%$ of patients. Clindamycin resistance was common, while rates of trimethoprim-sulfamethoxazole resistance were low. This study suggested the possibility of healthcare-associated transmission of CA-MRSA. This is the first Australian report of CA-MRSA superseding MSSA as the cause of community onset staphylococcal SSTI.

Key words: Staphylococcus aureus, Methicillin - S. aureus resistant to (MRSA), Public health microbiology, Soft tissue infections, Community epidemics.

\footnotetext{
* Author for correspondence: Eleanor MacMorran, Department of Microbiology and Infectious Diseases, John Radcliffe Hospital, Oxford, UK.

(Email: emacmorran@gmail.com, eleanor.macmorran@ouh.nhs. uk)
}

\section{INTRODUCTION}

Staphylococcus aureus is an important human pathogen associated with a variety of clinical presentations ranging from mild to life threatening $[1,2]$. Worldwide, $S$. aureus is the leading cause of skin and soft tissue infection (SSTI) [3]. Severe SSTI 
including extensive carbuncles are common in Central Australia, particularly within the Indigenous population [4]. Infections may progress to involve deep structures (e.g. bone and joints), despite prompt surgical debridement and antibiotic therapy.

Alice Springs Hospital (ASH) is the only referral hospital servicing Central Australia and has one of the highest rates of $S$. aureus blood stream infection in the world, with a mean annual incidence rate in Indigenous patients of 160.7/100000 [5]. Tong et al. reported a higher rate of $S$. aureus blood stream infections in Indigenous Australians compared with non-Indigenous Australians, but a lower 30-day mortality for the Indigenous patients - likely attributable to their younger age at the time of infection [6].

Recently, rising rates of MRSA have been reported locally [7], Australia-wide [8] and globally [9, 10]. This epidemiological shift has reflected the emergence and dissemination of non-multi-resistant strains of MRSA (nmMRSA), which are genetically and phenotypically distinct from multi-resistant MRSA isolates (mMRSA) [11]. While MRSA rates are increasing, currently most community onset staphylococcal disease in Australia is due to methicillin-susceptible $S$. aureus (MSSA) [8].

The original multi-resistant MRSA phenotype (mMRSA) was associated with hospital acquisition (HA-MRSA); affecting frail, older patients. This contrasts with reports on nmMRSA that affects younger and often otherwise healthy patients, typically acquired in the community (community-associated methicillin-resistant $S$. aureus (CA-MRSA)) [10]. Worldwide, Indigenous populations appear to have higher rates of CA-MRSA and in fact the first report of CA-MRSA within Australia was in an Indigenous population in the Kimberley region of Western Australia [12]. It is now recognised that HA-MRSA strains occasionally circulate within the community; while CA-MRSA is increasingly associated with nosocomial transmission [13].

Incision and drainage is well recognised to be the mainstay of treatment for purulent skin infections $[10,14]$, while the role of adjuvant antibiotics remains unclear [15]. Some researchers have reported lack of active antibiotic therapy (i.e. to which the organism tests sensitive in vitro) increases the risk of treatment failure $[1,16]$ while others have found no such association $[9,17]$. There is no consensus regarding the optimum treatment duration for staphylococcal SSTI, although emerging evidence suggests that short courses ( $\leqslant 7$ days) are appropriate in the majority of cases [18].

Very few studies have addressed staphylococcal skin infection in Central Australia [19, 20]. Our study aimed to characterise the epidemiology, clinical presentation, antibiotic susceptibility profile and outcomes of community-onset staphylococcal skin infection in our region. We were particularly interested to observe whether or not failure to receive active antibiotic therapy would impact on clinical outcome.

\section{METHODS}

\section{Setting}

ASH is a 186-bed teaching hospital in Central Australia. It is the sole referral hospital for an area of approximately 1.6 million $\mathrm{km}^{2}$, serving the urban region of Alice Springs, as well as numerous remote communities in the Northern Territory, Western Australia and South Australia [21]. Indigenous patients account for more than $80 \%$ of separations for ASH [22], although 2011 census data indicate that only $26.8 \%$ of the population of the Northern Territory is of Aboriginal or Torres Strait Islander origin [23].

\section{Patient population}

Adult and paediatric patients with community onset, purulent skin infections (i.e. abscesses, including furuncles and carbuncles) were prospectively identified over the 6-month study period (May-October 2014). Enrolment was restricted to those patients with infections requiring hospital admission, who were planned to have surgical debridement, and did not include patients with less severe, smaller abscesses managed within the Emergency Department. Consecutive patients were enrolled in the study if culture from either a superficial swab or a theatre sample yielded $S$. aureus. Infections involving deep structures (e.g. tendon, bone) at baseline were excluded; as were secondarily infected wounds; and polymicrobial infections such as pilonidal abscesses and diabetic foot infections.

\section{Data collection}

A standardised data collection tool was used to extract information from paper-based hospital records, as well as from an electronic health network linking the hospital system with community medical clinics. Not 
all patients were registered on this electronic system, which accounts for some of the missing follow-up data in our study.

Variables of interest included patient demographics, co-morbidities, clinical presentation, medical and surgical management, culture and susceptibility results, and outcome data at 30 days post-discharge. Systemic inflammatory response syndrome (SIRS) scores were calculated for adult patients according to observations from the time of admission, according to previously validated criteria [24].

\section{Laboratory methods and definitions}

Cultures and antibiotic susceptibility testing were performed by the Microbiology Department of ASH, in accordance with Clinical Laboratory and Standards Institute (CLSI) guidelines. Antibiotic susceptibility profiles were determined by Vitek testing (bioMerieux, version 7.01).

Oxacillin susceptible isolates were considered to be MSSA, and oxacillin-resistant isolates were considered to be MRSA. As per a previously described classification scheme, MRSA isolates were defined as non-multi-resistant (nmMRSA) if their phenotype demonstrated resistance to $\leqslant 2$ non-beta lactam antibiotics, and multi-resistant (mMRSA) if resistant to $\geqslant 3$ classes of non-beta lactam antibiotics (including clindamycin, erythromycin, trimethoprimsulfamethoxazole, tetracycline, ciprofloxacin and gentamicin); however no examples of this latter phenotype were identified [5]. Clindamycin resistance was detected via the Vitek GP card, which incorporates testing for inducible clindamycin resistance.

Antibiotic therapy was determined by the treating clinicians. Therapy was defined as 'active' if the isolate was reported as susceptible to the prescribed drug on in vitro testing.

\section{Outcomes}

The outcomes of interest were: (1) progression of infection to involve deep structures; (2) need for further surgical debridement; (3) unplanned re-presentation to hospital; (4) unplanned presentation to a General Practitioner (GP); (5) a requirement for an additional or unplanned course of antibiotics (all within 30 days of discharge) and (6) length of hospital stay. We also examined a combined end-point of any adverse outcome - comparing patients with no adverse outcome, to those with one or more of outcomes (1)-(5).
Outcomes were correlated with selected variables, including patient demographics (ethnicity, remote residence); age (child or adult); infecting strain phenotype (MSSA or nmMRSA); and antibiotic regimen (active or non-active).

\section{Statistical methods}

All analyses were conducted in $\mathrm{R}$ version 3.1 .2 ( $\mathrm{R}$ Core Team 2015). On univariate analysis, $P$-values for the differences between MSSA and nmMRSA were calculated using the Wilcoxon rank-sum test for continuous data, and Fisher's exact test for count data. On multivariate analysis, multiple imputation was performed via predictive mean matching. Adjusted odds ratios were estimated using logistic regression. Imputed estimates were combined using Rubin's rules. Statistical significance was defined by a $P$ value of $<0.05$.

\section{Ethics}

Ethics approval was granted by the Central Australian Human Research Ethics Committee (HREC-14-223). Individual patient consent was not required by the committee since the study was observational in nature and had no impact on clinical management.

\section{RESULTS}

\section{Baseline characteristics}

Baseline characteristics are summarised in Table 1. A total of 160 eligible patients were enrolled during the 6-month study period. The median age was 32 years (IQR $18 \cdot 0,46 \cdot 2$ ); $78 \%$ of patients were Indigenous; $42 \%$ lived in a remote community; and $60 \%$ were female. Thirty-eight of the patients $(24 \%)$ were children aged $\leqslant 16$ years.

The majority of $S$. aureus isolates $(96 / 160,60 \%)$ were methicillin-resistant and all these were CA-MRSA. The remainder of cases were MSSA (64/160, 40\%).

\section{Predictors for CA-MRSA vs. MSSA}

There was no significant difference in rates of CA-MRSA among adults compared with children $\leqslant 16$ years $(59 \%$ vs. $63 \%, P=0.707)$. On univariate analysis, predictors of CA-MRSA infection were prior isolation of CA-MRSA (OR 2.22, $P=0 \cdot 031$ ); hospitalisation within the last 6 months (OR 2.32, $P=0 \cdot 018$ ) and haemodialysis dependence (12 patients with 
Table 1. Purulent skin infection due to MSSA and CA-MRSA-baseline patient characteristics

\begin{tabular}{|c|c|c|c|c|c|c|c|}
\hline \multirow[b]{2}{*}{ Variable type } & \multirow{2}{*}{$\begin{array}{l}\text { Total } \\
(n=160) \\
\text { No. }(\%)\end{array}$} & \multirow{2}{*}{$\begin{array}{l}\text { CA-MRSA } \\
(n=96)\end{array}$} & \multirow{2}{*}{$\begin{array}{l}\text { MSSA } \\
(n=64)\end{array}$} & \multicolumn{4}{|c|}{ CA-MRSA vs. MSSA infection } \\
\hline & & & & $\begin{array}{l}\text { Univariate } \\
(\mathrm{OR}, 95 \% \mathrm{CI})\end{array}$ & $P$ value & $\begin{array}{l}\text { Multivariate } \\
(\mathrm{OR}, 95 \% \mathrm{CI})\end{array}$ & $P$ value \\
\hline \multicolumn{8}{|l|}{ Demographic trait } \\
\hline Age (median, years) & 32 & 32 & 32 & & & & \\
\hline Female & $96(60 \cdot 0)$ & $59(61 \cdot 5)$ & $37(57 \cdot 8)$ & $1 \cdot 16(0 \cdot 61-2 \cdot 22)$ & $0 \cdot 645$ & $1 \cdot 03(0 \cdot 48-2 \cdot 18)$ & $0 \cdot 942$ \\
\hline Indigenous & $124(78 \cdot 0)$ & $79(83 \cdot 2)$ & $45(70 \cdot 3)$ & $2 \cdot 08(0 \cdot 98-4 \cdot 50)$ & $0 \cdot 058$ & $2 \cdot 00(0 \cdot 73-5 \cdot 47)$ & $0 \cdot 176$ \\
\hline Remote residence & $67(41 \cdot 9)$ & $43(44 \cdot 8)$ & $24(37 \cdot 5)$ & $1 \cdot 35(0 \cdot 71-2 \cdot 60)$ & $0 \cdot 360$ & $1 \cdot 11(0 \cdot 52-2 \cdot 47)$ & $0 \cdot 793$ \\
\hline MRSA previously isolated & $50(31 \cdot 6)$ & $36(38 \cdot 3)$ & $14(21 \cdot 9)$ & $2 \cdot 22(1 \cdot 09-4 \cdot 68)$ & $\mathbf{0} \cdot 031$ & $1 \cdot 30(0 \cdot 56-3 \cdot 05)$ & $0 \cdot 542$ \\
\hline Hospitalised in past $6 / 12$ & $57(36 \cdot 1)$ & $41(43 \cdot 6)$ & $16(25 \cdot 0)$ & $2 \cdot 32(1 \cdot 17-4 \cdot 75)$ & $0 \cdot 018$ & $2 \cdot 34(0 \cdot 97-5 \cdot 67)$ & $0 \cdot 058$ \\
\hline \multicolumn{8}{|l|}{ Comorbidity } \\
\hline Obesity & $49(30 \cdot 8)$ & $29(30 \cdot 2)$ & $20(31 \cdot 7)$ & $0.93(0 \cdot 47-1 \cdot 86)$ & $0 \cdot 837$ & $1 \cdot 02(0 \cdot 43-2 \cdot 43)$ & $0 \cdot 963$ \\
\hline Diabetes & $61(38 \cdot 1)$ & $41(42 \cdot 7)$ & $20(31 \cdot 7)$ & $1 \cdot 64(0 \cdot 85-3 \cdot 23)$ & $0 \cdot 145$ & $1 \cdot 76(0 \cdot 59-5 \cdot 29)$ & $0 \cdot 308$ \\
\hline Chronic kidney disease & $34(21 \cdot 2)$ & $25(26 \cdot 0)$ & $9(14 \cdot 1)$ & $2 \cdot 15(0 \cdot 96-5 \cdot 22)$ & $0 \cdot 074$ & $1.97(0 \cdot 54-7 \cdot 19)$ & $0 \cdot 304$ \\
\hline Haemodialysis & $12(7 \cdot 5)$ & $12(12 \cdot 5)$ & $0(0 \cdot 0)$ & - & $0 \cdot 002$ & - & - \\
\hline Asthma & $12(7 \cdot 5)$ & $10(10 \cdot 4)$ & $2(3 \cdot 1)$ & $3 \cdot 60(0 \cdot 91-23 \cdot 99)$ & $0 \cdot 106$ & $3 \cdot 17(0 \cdot 67-14 \cdot 97)$ & $0 \cdot 143$ \\
\hline Chronic respiratory disease & $2(1 \cdot 2)$ & $1(1 \cdot 0)$ & $1(1 \cdot 6)$ & $0.66(0.03-16.97)$ & $0 \cdot 773$ & $0 \cdot 40(0 \cdot 03-5 \cdot 43)$ & $0 \cdot 487$ \\
\hline Harmful alcohol use & $27(16 \cdot 9)$ & $16(16 \cdot 7)$ & $11(17 \cdot 2)$ & $0 \cdot 96(0 \cdot 42-2 \cdot 29)$ & $0 \cdot 931$ & $0 \cdot 61(0 \cdot 22-1 \cdot 72)$ & $0 \cdot 346$ \\
\hline Ischaemic heart disease & $9(5 \cdot 6)$ & $7(7 \cdot 3)$ & $2(3 \cdot 1)$ & $2 \cdot 44(0 \cdot 57-16 \cdot 73)$ & $0 \cdot 276$ & $1 \cdot 14(0 \cdot 21-6 \cdot 25)$ & $0 \cdot 880$ \\
\hline Congestive cardiac failure & $10(6 \cdot 2)$ & $6(6 \cdot 2)$ & $4(6 \cdot 2)$ & $1 \cdot 00(0 \cdot 27-4 \cdot 05)$ & $1 \cdot 000$ & $0 \cdot 70(0 \cdot 15-3 \cdot 30)$ & $0 \cdot 654$ \\
\hline Scabies & $23(14 \cdot 4)$ & $14(14 \cdot 6)$ & $9(14 \cdot 1)$ & $1 \cdot 04(0 \cdot 43-2 \cdot 66)$ & $0 \cdot 927$ & $0 \cdot 62(0 \cdot 22-1 \cdot 78)$ & $0 \cdot 374$ \\
\hline
\end{tabular}

MSSA, methicillin-susceptible Staphylococcus aureus; CA-MRSA, community associated methicillin-resistant S. aureus; OR, odds ratio; CI, confidence interval.

Data are No. (\%) unless otherwise indicated.

CA-MRSA, 0 patients with MSSA; $P=0.002)$. On multivariate analysis, none of these factors reached statistical significance as predictors of CA-MRSA. There was a non-significant trend towards increased risk of CA-MRSA infection related to Indigenous status (OR $2 \cdot 08, P=0 \cdot 058)$.

\section{Clinical presentation and severity}

The median duration of symptoms prior to presentation was $5 \cdot 5$ days (IQR 3.0, 7.0), with a median abscess diameter of $5 \mathrm{~cm}$ (IQR $3 \cdot 0,6 \cdot 2$ ). Fifteen adult patients $(12 \cdot 5 \%)$ met the definition for SIRS. The decision about hospitalisation was based primarily on the local extensiveness of the abscess, and apparent need for formal surgical debridement under general anaesthetic. Clinical presentation did not differ with resistance phenotype (see Table 2).

\section{Antimicrobial susceptibility profile}

Differences in non-beta lactam antimicrobial susceptibilities were apparent between CA-MRSA and MSSA cases. The majority $(90 \cdot 6 \%)$ of CA-MRSA isolates were clindamycin sensitive, compared with only
$40 \cdot 6 \%$ of MSSA isolates. Trimethoprim-sulfamethoxazole (TMP-SMX) sensitivity was $100 \%$ for MSSA isolates, while 83 of $96(86 \%)$ of CA-MRSA isolates were TMP-SMX sensitive.

\section{Treatment details}

A surgical drainage procedure was performed in $99 \%$ of patients; with a median time to surgery of 1 day from time of admission.

All patients except one were commenced on empirical intravenous antibiotics. In $55.6 \%$ of patients, the antibiotics prescribed in the first $48 \mathrm{~h}$ post-admission were active against the subsequently isolated organism. Patients with CA-MRSA infection were less likely to receive an active antibiotic in the first $48 \mathrm{~h}$ compared with MSSA cases $(23.6 \%$ vs. $100 \%, P<$ $0 \cdot 001)$. After changing to oral therapy, treatment was active in $80.4 \%$ of patients; however, CA-MRSA cases were still less likely to receive an active agent compared with MSSA cases $(69 \cdot 0 \%$ vs. $98.2 \%, P<0 \cdot 001)$. Overall, the median planned oral antibiotic duration was 5 days. 
Table 2. Purulent skin infection due to MSSA and CA-MRSA - clinical presentation, treatment details and antibiotic susceptibility profile

\begin{tabular}{|c|c|c|c|c|}
\hline Variable type & $\begin{array}{l}\text { Total } \\
(n=160)\end{array}$ & $\begin{array}{l}\text { CA-MRSA } \\
(n=96)\end{array}$ & $\begin{array}{l}\text { MSSA } \\
(n=64)\end{array}$ & $P$ value \\
\hline \multicolumn{5}{|l|}{ Clinical presentation } \\
\hline Abscess size (diameter, $\mathrm{cm})$ & $5 \cdot 0$ & $5 \cdot 0$ & $4 \cdot 0$ & $0 \cdot 962$ \\
\hline Duration of symptoms pre-presentation (median, days) & $5 \cdot 5$ & $5 \cdot 0$ & $7 \cdot 0$ & $0 \cdot 959$ \\
\hline SIRS definition met (excluding children) & $15(12 \cdot 5)$ & $8(11 \cdot 4)$ & $7(14 \cdot 0)$ & $0 \cdot 782$ \\
\hline \multicolumn{5}{|l|}{ Treatment details } \\
\hline Surgical drainage performed & $158(98 \cdot 8)$ & $95(99 \cdot 0)$ & $63(98 \cdot 4)$ & $1 \cdot 000$ \\
\hline Time from admission to surgical drainage (median, days) & $1 \cdot 0$ & $1 \cdot 0$ & $1 \cdot 0$ & $0 \cdot 522$ \\
\hline Active IV antibiotics in initial $48 \mathrm{~h}$ & $85(55 \cdot 6)$ & $21(23 \cdot 6)$ & $64(100 \cdot 0)$ & $<0 \cdot 001$ \\
\hline Active oral antibiotics & $115(80 \cdot 4)$ & $60(69 \cdot 0)$ & $55(98 \cdot 2)$ & $<0 \cdot 001$ \\
\hline \multicolumn{5}{|l|}{ Non-beta lactam susceptibility profile } \\
\hline Clindamycin sensitive & $113(70 \cdot 6)$ & $87(90 \cdot 6)$ & $26(40 \cdot 6)$ & $<0 \cdot 001$ \\
\hline TMP-SMX sensitive & $147(91 \cdot 6)$ & $83(86 \cdot 5)$ & $64(100 \cdot 0)$ & $0 \cdot 002$ \\
\hline
\end{tabular}

MSSA, methicillin-susceptible Staphylococcus aureus; CA-MRSA, community associated methicillin-resistant S. aureus; SIRS, systemic inflammatory response syndrome; TMP-SMX, trimethoprim-sulfamethoxazole.

Data are No. (\%) unless otherwise indicated.

\section{Outcomes}

Within 30 days of discharge, $13 \cdot 5 \%$ of patients had an un-planned representation to hospital with the same complaint, while $19 \cdot 7 \%$ had an unplanned GP presentation. The most serious adverse outcomes were considered to be a requirement for further debridement in theatre $(9 \cdot 6 \%$ of patients) and progression of infection to involve deep structures (5.2\% of patients). It was common for additional courses of antibiotics to be prescribed $(27 \cdot 8 \%$ of patients). There was one death within 30 days of discharge (believed to be from an unrelated cause). Overall, 39\% of patients experienced at least one adverse outcome. More adults than children experienced at least one adverse outcome $(41.8 \%$ vs. $28.9 \%)$, however this difference was not statistically significant $(P=0 \cdot 184)$ and did not differ with receipt or not of active antibiotic therapy (35.9\% vs. $42 \cdot 7 \%, P=0 \cdot 415$ ).

There was a high rate of missing data regarding progression to deep structures, unplanned GP presentation and repeat antibiotics $(\sim 22 \%)$ but only a minimal amount $(\sim 2 \%)$ for representation to hospital; repeat debridement in theatre; and length of stay. The median length of hospital stay overall was 3 days (IQR 2.0, 5.5) with CA-MRSA patients having a shorter length of stay compared with MSSA patients (rate ratio $0.78, P<0.001$ ); however, this result was not significant on multivariate analysis $(P=0 \cdot 116)$. Indigenous patients had a longer length of stay relative to non-Indigenous patients, as did those living remotely (see Table 3). On multivariate analysis, increased length of hospital stay also correlated with the presence of diabetes (rate ratio $1.57, P=0.001$ ); congestive cardiac failure (rate ratio $3 \cdot 12, P<0 \cdot 001$ ); and chronic respiratory disease $(1 \cdot 78, P=0 \cdot 018)$.

\section{DISCUSSION}

This is the first time that CA-MRSA has been reported as the dominant phenotype in an Australian population. Causing $60 \%$ of cases, CA-MRSA has now superseded MSSA as a cause of community onset purulent SSTI in Central Australia.

More broadly, it is conceivable that the ascendency of CA-MRSA observed here may foreshadow a more widespread ecological shift across Australia. Tong et al. previously raised the concern that Australian Indigenous communities may act as foci for the emergence of CA-MRSA, in a manner analogous to the US prison populations that were thought to represent core transmitters of the USA300 clone [25]. When considering the epidemiology of $S$. aureus in Central Australia, it is helpful to compare with the experiences of the Northern Territory's 'Top End', since both these regions contain urban and remote populations with high rates of social disadvantage $[4,19]$. A recent Top End study described a threefold rise in community-acquired MRSA infections, from $7 \%$ to $24 \%$ over the course of the last two decades [7]. This compares with a 2012 nationwide survey in 
Table 3. Purulent skin infection due to MSSA and CA-MRSA - study outcomes

\begin{tabular}{|c|c|c|c|c|c|c|c|c|c|c|c|}
\hline \multicolumn{2}{|c|}{$\begin{array}{l}\text { Progression of infection to deep } \\
\text { structures }\end{array}$} & \multicolumn{2}{|c|}{$\begin{array}{l}\text { Need for further surgical } \\
\text { debridement }\end{array}$} & \multicolumn{2}{|c|}{$\begin{array}{l}\text { Unplanned hospital } \\
\text { re-presentation }\end{array}$} & \multicolumn{2}{|c|}{ Unplanned GP presentation } & \multicolumn{2}{|c|}{$\begin{array}{l}\text { Requirement for additional } \\
\text { antibiotics }\end{array}$} & \multicolumn{2}{|c|}{ Length of hospital stay } \\
\hline \multicolumn{12}{|l|}{ OR, $95 \% \mathrm{CI}$} \\
\hline Univariate & Multivariate & Univariate & Multivariate & Univariate & Multivariate & Univariate & Multivariate & Univariate & Multivariate & Univariate & Multivariate \\
\hline \multicolumn{12}{|l|}{ Female } \\
\hline $0 \cdot 92(0 \cdot 20,4 \cdot 85)$ & $0.94(0 \cdot 13-7 \cdot 09)$ & $0 \cdot 22(0 \cdot 06-0 \cdot 67)$ & $0 \cdot 12(0 \cdot 02-0 \cdot 67)$ & $0.57(0.22-1 \cdot 44)$ & $0 \cdot 66(0 \cdot 18-2 \cdot 50)$ & $2 \cdot 04(0 \cdot 77-6 \cdot 04)$ & $1.94(0.54-6.93)$ & $0.82(0 \cdot 36-1 \cdot 86)$ & $0.59(0 \cdot 19-1 \cdot 83)$ & $1 \cdot 09(0 \cdot 96-1 \cdot 25)$ & $0.87(0.72-1.04)$ \\
\hline$P 0.533$ & $P 0.954$ & $P 0.012$ & $P 0.016$ & $P 0.232$ & $P 0.542$ & $P 0 \cdot 168$ & $P 0.304$ & $P 0.623$ & $P 0.356$ & $P 0 \cdot 183$ & $P 0 \cdot 119$ \\
\hline \multicolumn{12}{|l|}{ Indigenous } \\
\hline $0.58(0 \cdot 12-4 \cdot 24)$ & $0 \cdot 28(0.02-4 \cdot 48)$ & $1.91(0.49-12.61)$ & $1 \cdot 58(0 \cdot 18-13 \cdot 61)$ & $0 \cdot 48(0 \cdot 18-1 \cdot 38)$ & $0 \cdot 28(0 \cdot 05-1 \cdot 60)$ & $3 \cdot 37(0 \cdot 61-62 \cdot 91)$ & $2 \cdot 10(0 \cdot 24-18 \cdot 27)$ & $1.30(0 \cdot 42-4.90)$ & $0.73(0.13-4.01)$ & $2 \cdot 87(2 \cdot 31-3 \cdot 62)$ & $1.59(1.21-2.09)$ \\
\hline$P 0.533$ & $P 0.366$ & $P 0.411$ & $P 0.673$ & $P 0.153$ & $P 0 \cdot 151$ & $P 0.255$ & $P 0.490$ & $P 0.688$ & $P 0.713$ & $P<0.001$ & $P 0.001$ \\
\hline \multicolumn{12}{|c|}{ Remote residence } \\
\hline $1 \cdot 86(0 \cdot 39-9 \cdot 78)$ & $1.92(0.23-15 \cdot 77)$ & $1 \cdot 66(0 \cdot 56-4 \cdot 96)$ & $1 \cdot 36(0 \cdot 30-6 \cdot 14)$ & $0 \cdot 38(0 \cdot 12-1 \cdot 03)$ & $0 \cdot 24(0 \cdot 05-1 \cdot 06)$ & $1.60(0.65-3.99)$ & $1 \cdot 06(0 \cdot 28-3.94)$ & $0.99(0 \cdot 44-2 \cdot 20)$ & $0.50(0 \cdot 17-1 \cdot 42)$ & $1.50(1.32-1 \cdot 71)$ & $1.39(1.17-1.65)$ \\
\hline$P 0.428$ & $P 0 \cdot 540$ & $P 0.355$ & $P 0.693$ & $P 0.073$ & $P 0.060$ & $P 0.310$ & $P 0.932$ & $P 0.984$ & $P 0 \cdot 188$ & $P<0.001$ & $P<0.001$ \\
\hline \multicolumn{12}{|c|}{ CA-MRSA ( $v$. MSSA) } \\
\hline $1.62(0 \cdot 34-11 \cdot 6)$ & $3 \cdot 00(0 \cdot 17-52 \cdot 38)$ & $2 \cdot 91(0 \cdot 88-13 \cdot 2)$ & $3 \cdot 60(0 \cdot 36-36 \cdot 22)$ & $1.08(0.43-2.90)$ & $1 \cdot 46(0 \cdot 22-9 \cdot 57)$ & $1.44(0.56-4.04)$ & $3.99(0 \cdot 32-49 \cdot 88)$ & $1 \cdot 05(0 \cdot 46-2 \cdot 45)$ & $1 \cdot 19(0 \cdot 21-6 \cdot 63)$ & $0.78(0.69-0.89)$ & $0.74(0.54-1.02)$ \\
\hline$P 0.571$ & $P 0 \cdot 448$ & $P 0 \cdot 108$ & $P 0.277$ & $P 0.868$ & $P 0.693$ & $P 0.463$ & $P 0.275$ & $P 0.912$ & $P 0.841$ & $P<0.001$ & $P 0.069$ \\
\hline \multicolumn{12}{|c|}{ Active IV antibiotics-1st $48 \mathrm{~h}$} \\
\hline $0.62(0 \cdot 12-2 \cdot 91)$ & $1.99(0 \cdot 18-22.66)$ & $0 \cdot 40(0 \cdot 12-1 \cdot 22)$ & $0 \cdot 85(0 \cdot 12-6 \cdot 21)$ & $2.03(0.76-6.03)$ & $2 \cdot 47(0 \cdot 40-15 \cdot 24)$ & $0.94(0.35-2 \cdot 49)$ & $3 \cdot 26(0 \cdot 23-46 \cdot 75)$ & $1 \cdot 11(0 \cdot 49-2 \cdot 52)$ & $2.58(0.53-12.52)$ & $1 \cdot 26(1 \cdot 10-1 \cdot 44)$ & $0.86(0.63-1 \cdot 17)$ \\
\hline$P 0.537$ & $P 0.575$ & $P 0 \cdot 118$ & $P 0.871$ & $P 0.173$ & $P 0.325$ & $P 0.894$ & $P 0.370$ & $P 0.808$ & $P 0.237$ & $P 0.001$ & $P 0.339$ \\
\hline \multicolumn{12}{|c|}{ Active oral antibiotics } \\
\hline $1 \cdot 44(0 \cdot 22-28 \cdot 2)$ & $0.82(0 \cdot 04-15 \cdot 18)$ & N/A & $0 \cdot 85(0 \cdot 12-6 \cdot 21)$ & $1 \cdot 14(0 \cdot 34-5 \cdot 24)$ & $1 \cdot 79(0 \cdot 25-13 \cdot 00)$ & $0.58(0 \cdot 20-1 \cdot 85)$ & $0.57(0.09-3.60)$ & $0.78(0 \cdot 30-2 \cdot 14)$ & $0.52(0 \cdot 12-2 \cdot 27)$ & $2.06(1.66-2.59)$ & $1.47(1.04-2.09)$ \\
\hline$P 0.746$ & $P 0.894$ & N/A & $P 0.871$ & $P 0.843$ & $P 0.562$ & $P 0.334$ & $P 0.544$ & $P 0.615$ & $P 0.378$ & $P<0.001$ & $P 0.031$ \\
\hline
\end{tabular}

MSSA, methicillin-susceptible Staphylococcus aureus; CA-MRSA, community associated methicillin-resistant $S$. aureus; OR, odds ratio; CI, confidence interval; GP, General Practitioner. 
Australia that reported CA-MRSA accounting for $12.5 \%$ of all $S$. aureus isolates (SSTI specimens comprised $90 \cdot 5 \%$ of all specimens) [8]. The rate of $60 \%$ CA-MRSA in our study is of great concern, and appears to be on an upward trajectory.

The current Central Australian experience resembles the emergence of CA-MRSA skin infection observed in the USA over recent years $[9,26]$. While the US epidemic is largely attributable to the rapid rise of USA300 as a single virulent clone, the molecular epidemiology of $S$. aureus within Australia is more complex. The increase in CA-MRSA infections observed throughout Australia reflects the emergence of multiple clones [4, 10, 27]. An analysis of $S$. aureus SSTI isolates from Central Australia revealed a high proportion of panton valentine leucocidin (PVL) across both MSSA and CA-MRSA. Whilst ST93 CA-MRSA and CC121 MSSA predominated, there was wide variety of circulating clones [28]. It has been postulated that CA-MRSA clones in the Top End have arisen de novo in the community, as a consequence of circulating MSSA strains independently acquiring SCC mecIV from sources such as coagulase negative staphylococci $[4,25]$.

The process of SCC mecIV acquisition may be facilitated by the high organism burdens that arise when living conditions are crowded and access to the basics required for hygiene is limited, as is common in remote Indigenous communities $[4,7,25,29]$. The frequent use of beta lactam antibiotics in these communities may contribute towards persistence of the methicillin-resistant phenotype $[4,7,30]$.

We found a disproportionate burden of staphylococcal skin disease among Indigenous patients. Indigenous people comprise approximately one quarter of the population in this region [23], but accounted for more than three quarters of infections in this study. A similar trend was previously reported with $S$. aureus bacteraemia in Central Australia [5]. A trend towards higher risk of CA-MRSA amongst Indigenous patients (univariate OR 2.08, 95\%; CI 0.98-4.50; $P$ $=0.058$ ) was also reported in a recent local study of blood stream infections [19] and a multi-centre prospective Australian study [6]. However, it can be difficult to distinguish the impact of ethnicity from potentially confounding variables such as the high chronic disease prevalence (including diabetes and haemodialysis dependence) within Indigenous populations, which may be independently associated with increased infection risk [19].
A notable finding of this study was that both hospitalisation within the previous 6 months and haemodialysis dependence appeared to be predictors for CA-MRSA infection relative to MSSA infection, suggesting possible health-care associated transmission. Within Australia, CA-MRSA has not typically been considered a nosocomial pathogen, however, a study from the Royal Darwin Hospital reported that inpatients were 14 times more likely to be colonised with CA-MRSA compared with their status at the time of admission [31]. Internationally, the epidemiology also appears to be changing in this regard, with an increasing number of studies reporting that CA-MRSA is now being transmitted from the community back into hospitals $[15,32]$. It is apparent from the literature that dialysis patients are at increased risk of $S$. aureus blood stream infections; as well as being at increased risk for MRSA colonisation and infection [33] although much of this data refers to mMRSA rather than the nmMRSA seen in our study.

Some international reports have suggested more severe skin disease associated with CA-MRSA, particularly in relation to the methicillin resistant USA300 clone [34, 35]. However our study did not demonstrate any correlation of $S$. aureus resistance profile with either clinical presentation or with study outcomes, and aligns with reports from the neighbouring 'Top End' [4] of Australia.

In our study, $39 \%$ of patients overall experienced at least one adverse outcome, which is higher than has previously described in primary care based studies that reported failure rates of between 10\% [36] and $21 \%$ [37]. This discrepancy may be explainable by our chosen study population (hospitalised patients, rather than those managed in the primary care setting), but our study does raise questions about whether particular virulence characteristics might exist among the $S$. aureus clones circulating in this region. The association we found between the presence of certain co-morbidities (e.g. diabetes) and increased length of hospital stay could potentially be a reflection of impaired healing; or the need for expanded care while in hospital.

Receipt of an active antibiotic was associated with a longer length of hospital stay, perhaps due to reverse causality - additional time for drug susceptibility results to become available and be acted upon appropriately by clinicians. Otherwise, antibiotic choice did not affect outcomes in our study, probably because almost all patients underwent formal surgical 
debridement in theatre. Lack of a drainage procedure is strongly associated with increased risk of clinical failure $[9,16]$ and there is some evidence to indicate that drainage alone is often sufficient to achieve cure, irrespective of concurrent antibiotic therapy $[10,34,38]$. However, contrary results have been reported by other authors who suggest concurrent antibiotics are required [16, 39].

An unexpected finding of our study was a high rate of lincosamide resistance $-59 \cdot 4 \%$ of MSSA and $9 \cdot 4 \%$ of CA-MRSA isolates were resistant to clindamycin. Molecular typing suggests that differences correlate with clonal complex type, with $\mathrm{CC} 121$ (primarily MSSA) commonly clindamycin resistant $(60 \%)$; and ST93 (primarily CA-MRSA) infrequently clindamycin resistant $(12 \cdot 5 \%)$ [28].

This degree of clindamycin resistance limits its utility as an empiric antibiotic choice. Given the shared resistance mechanism for lincosamides and macrolides [40] this phenomenon may be linked to the widespread use of macrolides in our region [7]. TMP-SMX appears to be a more reliable choice as an empiric oral treatment option for staphylococcal SSTI in this population.

Our study has several limitations. Its small sample size limits its power to detect differences among the outcome subgroups. We have only included strains and data from a subgroup of SSTI, namely those presenting with abscesses requiring surgical drainage. Given the limited sampling time frame, these data provide a time limited snapshot and may not be generalisable to a broader time period, nor do they provide a longitudinal view. Most notably, the clinicians' choice of whether or not to include an MRSA active agent as part of empiric therapy is likely to have been a weighted decision. It may be that those patients with more severe infections had a greater likelihood of receiving active therapy. Consequently, the negative effect of more severe disease at baseline may have diluted any potential benefit to be gained by active empiric therapy.

\section{CONCLUSION}

To our knowledge, this is the first time that rates of CA-MRSA infection have exceeded MSSA in the Australian literature, and this may well be a signal of future changes in the epidemiology of $S$. aureus in Australia. Locally, in light of such extreme rates of CA-MRSA, empiric antibiotic selection for SSTI in this population should routinely include CA-MRSA cover, particularly in the context of sepsis.

We were not able to demonstrate an association between antimicrobial therapy (active $v s$. non-active) and clinical outcome. Unlike many other reports within the literature, our study cohort underwent formal surgical debridement, rather than incision and drainage in the Emergency Department or out-patient setting. The contribution of antibiotic therapy to cure is likely to be relatively less important when definitive source control has been achieved by surgery.

Public health strategies which address the issues of inadequate housing and poverty are likely to have the greatest impact in terms of reducing the burden of staphylococcal skin disease; as well as limiting the progression of CA-MRSA in Australia.

\section{ACKNOWLEDGEMENTS}

James McLeod and the Microbiology Department, Alice Springs Hospital (ASH). Mr Jacob Jacobs and the Department of Surgery, ASH.

\section{DECLARATION OF INTEREST}

None.

\section{REFERENCES}

1. Ruhe JJ, et al. Community-onset methicillin-resistant Staphylococcus aureus skin and soft-tissue infections: impact of antimicrobial therapy on outcome. Clinical Infectious Diseases 2007; 44: 777-784.

2. Tong SY, et al. Staphylococcus aureus infections: epidemiology, pathophysiology, clinical manifestations, and management. Clinical Microbiology Reviews 2015; 28: $603-661$.

3. Gould IM. Antibiotics, skin and soft tissue infection and methicillin-resistant Staphylococcus aureus: cause and effect. International Journal of Antimicrobial Agents 2009; 34: 58-61.

4. Tong SY, et al. Community-associated strains of methicillin resistant Staphylococcus aureus and methicillin susceptible S. aureus in Indigenous Northern Australia: epidemiology and outcomes. Journal of Infectious Diseases 2009; 199: 1461-1470.

5. Hewagama S, Spelman T, Einsiedel LJ. Staphylococcus aureus bacteraemia at Alice Springs Hospital, Central Australia, 2003-2006. Internal Medicine Journal 2012; 42: 505-512.

6. Tong SY, et al. Impact of ethnicity and socio-economic status on Staphylococcus aureus bacteremia incidence and mortality: a heavy burden in Indigenous Australians. BMC Infectious Diseases 2012; 12: 249. 
7. Tong SY, et al. Progressive increase in community-associated methicillin resistant Staphylococcus aureus in Indigenous populations in northern Australia from 1993 to 2012. Epidemiology and Infection 2015; 143: 1519-1523.

8. Coombs GW, et al. for the Australian Group on Antimicrobial Resistance (AGAR). Community-onset Staphylococcus aureus surveillance programme annual report, 2012. Communicable Diseases Intelligence 2014; 38: E59-E69.

9. Miller LG, et al. A prospective investigation of outcomes after hospital discharge for endemic community-acquired methicillin-resistant and -susceptible Staphylococcus aureus skin infection. Clinical Infectious Diseases 2007; 44: 483-492.

10. Chua K, et al. Not community-associated methicillinresistant Staphylococcus aureus (CA-MRSA)! A clinician's guide to community MRSA - its evolving antimicrobial resistance and implications for therapy. Clinical Infectious Diseases 2011; 52: 99-114.

11. Ng JW, et al. Phylogenetically distinct Staphylococcus aureus lineage prevalent among Indigenous communities in Northern Australia. Journal of Clinical Microbiology 2009; 47: 2295-2300.

12. Udo EE, Pearman JW, Grubb WB. Genetic analysis of community isolates of methicillin-resistant Staphylococcus aureus in Western Australia. Journal of Hospital Infection 1993; 25: 97-108.

13. Seybold U, et al. Emergence of community-associated methicillin resistant Staphylococcus aureus USA300 genotype as a major cause of health care-associated blood stream infections. Clinical Infectious Diseases 2006; 42: 647-656.

14. Williams DJ, et al. Comparative effectiveness of antibiotic treatment strategies for pediatric skin and softtissue infections. Pediatrics 2011; 128: e479-e487.

15. Stryjewski ME, Chambers HF. Skin and soft-tissue infections caused by community-acquired methicillinresistant Staphylococcus aureus. Clinical Infectious Diseases 2008; 46: S368-S377.

16. Frei CR, et al. Trimethoprim-sulfamethoxazole or clindamycin for community-associated MRSA (CA-MRSA) skin infections. Journal of the American Board of Family Medicine 2010; 23: 714-719.

17. Chen AE, et al. Randomised controlled trial of cephalexin versus clindamycin for uncomplicated pediatric skin infections. Pediatrics 2011; 127: e573-e580.

18. Schuler CL, et al. Decreasing duration of antibiotic prescribing for uncomplicated skin and soft tissue infections. Pediatrics 2016; 137: 1-7.

19. Einsiedel L, et al. Non-communicable diseases, infection and survival in a retrospective cohort of Indigenous and non-Indigenous adults in central Australia. British Medical Journal Open 2013; 3: e003070.

20. Stevens CL, et al. Community-acquired methicillinresistant Staphylococcus aureus in Central Australia. Communicable Diseases Intelligence 2006; 30: 462-466.

21. Northern Territory Government Department of Health. Alice Springs Hospital 2016. Retrieved from http://www. health.nt.gov.au/Hospitals/Alice_Springs_Hospital/index. aspx.
22. Northern Territory Government Department of Health. Annual Report 2013-14. Retrieved from http://www. health.nt.gov.au/Publications/Corporate_Publications/ index.aspx.

23. Australian Bureau of Statistics 2011. Census of Population and Housing - Counts of Aboriginal and Torres Strait Islander Australians. Cat no. 2075.0, ABS, Canberra.

24. American College of Chest Physicians/Society of Critical Care Medicine Consensus Conference: definitions for sepsis and organ failure and guidelines for the use of innovative therapies in sepsis. Critical Care Medicine 1992; 20: 864-874.

25. Tong SY, et al. Global implications of the emergence of community-associated methicillin-resistant Staphylococcus aureus in Indigenous populations. Clinical Infectious Diseases 2008; 46: 1871-1878.

26. Talan DA, et al. Comparison of Staphylococcus aureus from skin and soft-tissue infections in US emergency department patients, 2004 and 2008. Clinical Infectious Diseases 2011; 53: 144-149.

27. Stinear TB, et al. Adaptive change inferred from genomic population analysis of the ST93 epidemic clone of community-associated methicillin-resistant Staphylococcus aureus. Genome Biology and Evolution 2014; 6: 366-378.

28. Harch SA, et al. High burden of complicated skin and soft tissue infections in the Indigenous population of Central Australia due to dominant Panton Valentine Leucocidin clones ST93-MRSA and CC121-MSSA. BMC Infectious Diseases 2017; 17: 405.

29. McDonald M, et al. Use of a single-nucleotide polymorphism genotyping system to demonstrate the unique epidemiology of methicillin-resistant Staphylococcus aureus in remote Aboriginal communities. Journal of Clinical Microbiology 2006; 44: 3720-3727.

30. Baggett HC, et al. Community-onset methicillinresistant Staphylococcus aureus associated with antibiotic use and the cytotoxin Panton-Valentine leucocidin during a furunculosis outbreak in rural Alaska. Journal of Infectious Diseases 2004; 189: 1565-1573.

31. Brennan L, et al. Community-associated methicillinresistant Staphylococcus aureus carriage in hospitalized patients in tropical Northern Australia. Journal of Hospital Infection 2013; 83: 205-211.

32. O'Brien FG, et al. Population dynamics of methicillinsusceptible and -resistant Staphylococcus aureus in remote communities. Journal of Antimicrobial Chemotherapy 2009; 64: 684-693.

33. Zacharioudakis IM, et al. Meta-analysis of methicillinresistant Staphylococcus aureus colonization and risk of infection in dialysis patients. Journal of the American Society of Nephrology 2014; 25: 2131-2141.

34. Jahamy H, et al. Staphylococcus aureus skin/soft-tissue infections: the impact of SCCmec type and Panton-Valentine leucocidin. Scandinavian Journal of Infectious Diseases 2008; 40: 601-606.

35. Young DM, et al. An epidemic of methicillin-resistant Staphylococcus aureus soft tissue infections among 
medically underserved patients. Archives of Surgery 2004; 139: 947-953.

36. May LS, et al. Treatment failure outcomes for emergency department patients with skin and soft tissue infections. Western Journal of Emergency Medicine 2015; 5: 642-652.

37. Lee GC, et al. A prospective observational cohort study in primary care practices to identify factors associated with treatment failure in Staphylococcus aureus skin and soft tissue infections. Annals of Clinical Microbiology and Antimicrobials 2016; 15: 1-7.
38. O'Brien FG, et al. Diversity among community isolates of methicillin-resistant Staphylococcus aureus in Australia. Journal of Clinical Microbiology 2004; 42: 3185-3190.

39. Talan DA, et al. Trimethoprim-Sulfamethoxazole versus placebo for uncomplicated skin abscess. New England Journal of Medicine 2016; 374: 823-832.

40. Leclercq R. Mechanisms of resistance to macrolides and lincosamides: nature of resistance elements and their clinical implications. Clinical Infectious Diseases 2002; 34: 482-492. 